\title{
LIGHT ENVIRONMENT AND LAI MONITORING IN RICE COMMUNITY BY GROUND- AND UAV-BASED REMOTE SENSING
}

\author{
M. Yamashita ${ }^{1 *}$, H. Toyoda ${ }^{2}$, Y. Tanaka ${ }^{2}$ \\ ${ }^{1}$ Institute of Agriculture, Tokyo University of Agriculture and Technology, Fuchu, Tokyo, Japan - meguyama@cc.tuat.ac.jp \\ ${ }^{2}$ Graduate School of Agriculture, Tokyo University of Agriculture and Technology, Fuchu, Tokyo, Japan
}

Commission III, WG III/10

KEY WORDS: light environment, LAI, vegetation color index, vegetation coverage, plant height

\begin{abstract}
:
Cultivation management adapted to various environmental changes has been required under changing climate, hence it is necessary to monitor the growth status efficiently. Photosynthesis in crop production is the essential process in crop growth model for forecasting the yield. In this study, we focused on light environment and LAI as important factors in photosynthesis, and conducted the observations to obtain time-series RGB images taken by digital cameras fixed on tower and mounted on UAV in the paddy field and to measure PPFDs at the top ( $\left.\mathrm{I}_{0}\right)$ and the bottom (I) of rice canopy during planted period. Three kinds of vegetation color indices (CIs), vegetation coverage (VC) and plant height $(\mathrm{PH})$ derived by processing RGB images were compared with relative PPFD (I/I0) and LAI calculated from relative PPFD. As the results, CIs and VC were basically expressed linear relations with relative PPFD and PH showed the exponential relation with relative PPFD and the linear relation with LAI respectively. Through this study, we clarified that VC and PH would be effective parameters for spatio-temporal monitoring of light environment and LAI.
\end{abstract}

\section{INTRODUCTION}

Abnormal and extreme weather conditions have significantly changed the growth environment during the growing process of crops, and have a significant effect on stable crop production. Cultivation management adapted to various environmental changes under changing climate is required more than ever, and it is indispensable to monitor the growth status of crops and predict the yield. Photosynthesis in crop production is one of the essential process in crop growth model and forecasting the yield. However, it is difficult to measure leaf area index (LAI) and the light environment within the canopy, which are important factors in canopy photosynthetic model, continuously and spatially.

Satellite remote sensing can monitor crop fields widely, but, there is sometimes limitation to analyze images depending on the purpose of use (ex: spatial resolution, small size field etc.) (Cen et al., 2019). In recent years, with the spread of unmanned aerial vehicles (UAVs), smart agriculture utilizing UAVs, which can observe from low altitude, has attracted attention (Aasen et al., 2018; Honkavaara et al., 2019; Li et al., 2019; Maes, Steppe, 2019). Since the crop growth situations has been affected by the cultivation environment, especially the weather, it is also need to observe them continuously and efficiently at short intervals.

In this study, we focus on the light environment and LAI in rice community at the field scale in order to develop the spatiotemporal model of crop canopy photosynthesis in near future. The purpose of this study is to clarify the relationships between remotely sensed parameters and LAI / light environment within the canopy. For this purpose, we obtained time-series RGB images using a fixed point camera and UAV and measured PPFDs at the top ( $\left.\mathrm{I}_{0}\right)$ and the bottom (I) of the canopy in paddy field. We investigated each relation of relative PPFD and LAI with vegetation color indices, vegetation coverage and plant height derived from obtained RGB images.

\section{MATERIALS AND METHODS}

\subsection{Study site}

The study site was an experimental paddy field of Tokyo University of Agriculture and Technology (N35.6656, E139.4725) in Tokyo, Japan. The target crop variety was a rice (Nipponbare, Oryza sativa). Transplantation was performed at every $15 \mathrm{~cm}$ interval between the plants and $30 \mathrm{~cm}$ interval between the lines in the area of $6 \mathrm{~m}$ by $10 \mathrm{~m}$ on $22^{\text {nd }}$ May, 2019.

An observational tower with $3 \mathrm{~m}$ height and $10 \mathrm{~m}$ arm-length was built to fix the digital camera and the other sensors in this site (Figure 1). Four piles of ground control points (GCP) were installed at four corners of planted area to generate the digital surface model (DSM) from overlapped aerial images taken by UAV.

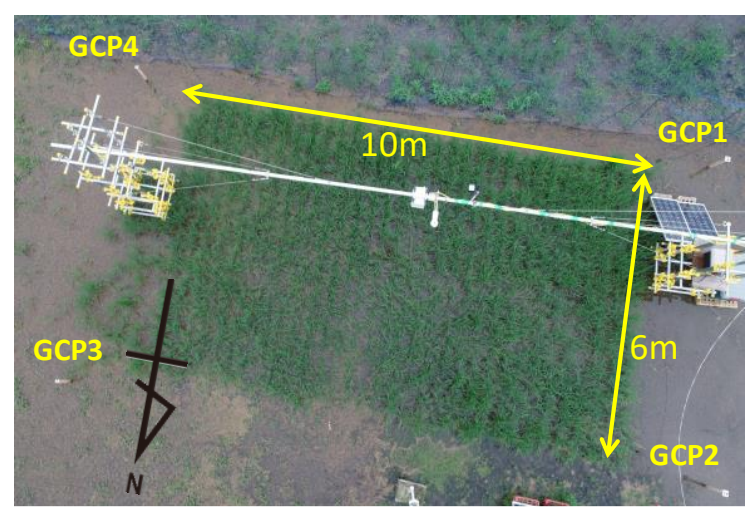

Figure 1. Study site and observational tower

\footnotetext{
* Corresponding author
} 


\subsection{Vegetation color indices and vegetation coverage}

A digital camera (Nikon D5300, Nikon) was used to capture images for calculating various vegetation color indices (CIs) and vegetation coverage $(V C)$. The camera was installed at nadir direction on a tower about $3 \mathrm{~m}$ height. The capturing period was from $14^{\text {th }}$ June to $23^{\text {rd }}$ September, 2019, and a total of about 3,000 images were taken at every 10 minutes. In order to reduce the influence of different color balance and brightness by different weather conditions, all taking images were normalized by liner conversion using the maximum RGB values (digital number of each red, green, blue) of the grayscale panel put in the field (Yamashita et al., 2019). We used the images taken from 9:00 to 15:00 each day to avoid the shadow effect and calculated green ratio (gratio) (Shimojima et al., 2017), normalized green and red difference (VIgreen) (Gitelson et al., 2002) and simple ratio of green to red $(G R)$ (Wang et al. 2014) as CIs by eq. 1-3 using the averaged $\mathrm{R}, \mathrm{G}$, and $\mathrm{B}$ values in the region of interest (ROI). ROI in the image was set about $1.6 \mathrm{~m}$ square including about 50 plants.

$$
\begin{gathered}
\text { gratio }=\frac{G}{B+G+R} \\
\text { VI green } \\
=\frac{G-R}{G+R} \\
G R=\frac{G}{R}
\end{gathered}
$$

$V C$ was calculated as the ratio of green leaves area in ROI. As for the detection of green leaves, we separated all pixels into green area and the other area by using CIE $L^{*} a^{*} b^{*}$ which were computed from tristimulus values $X, Y$, and $Z$. The green area was segmented using threshold of $a^{*}$ value for each image.

The calculated color index and detected green leaves area from RGB image are shown in Figure 2 for example.

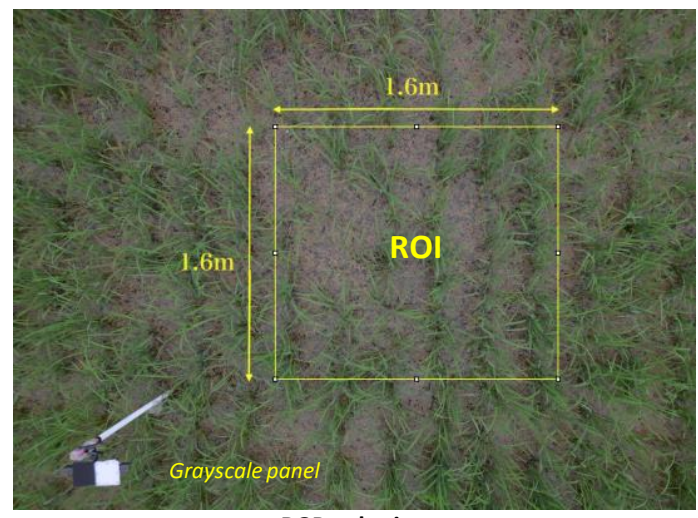

RGB color image

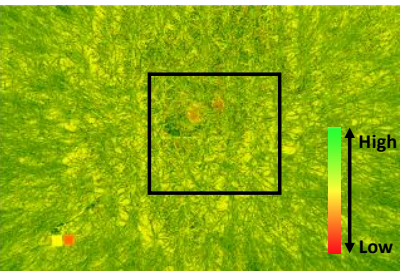

Color index (ex. gratio)

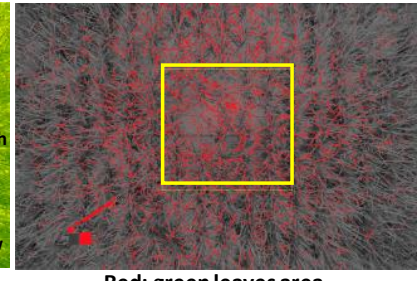

Red: green leaves area
Figure 2. RGB image, color index and green leaves area

\subsection{Plant height observation}

UAV (Inspire 2, DJI) with attached camera was used to capture aerial images for deriving plant height $(P H)$. We conducted trilateral surveying and levelling to fix the $x y z$-coordinate of four GCPs (Figure 1) by measuring the horizontal distance of each side and the vertical distance from ground at each point. The origin of the relative $x y z$-coordinate was set at southwest point (GCP1) on the ground and the horizontal axis was defined as the line from GCP 1 to GCP 4.

UAV aerial images were taken with about $80 \%$ overlap on total 13 times every week (about 60 images $/$ time) from $4^{\text {th }}$ June to $27^{\text {th }}$ August, 2019. We processed overlapped aerial images to generate DSM as follows: 1) tie points were automatically detected by image matching, 2) the parameters of camera calibration were derived, 3) four GCPs were manually fixed on each image, 4) the parameters of exterior orientation were estimated, 5) each GCP accuracy was checked, and 6) dense point clouds and meshes were built by using Metashape Professional (Agisoft). In this case, we could directly use $z$-values of DSM as the canopy surface model (CSM) to derive $P H$ in ROI.

\subsection{Light environment observation in rice community}

A total of six quantum sensors with small memory (DEFI2-L, JFE Advantech) for the observation of light environment in the rice community were installed at five places of the canopy bottom (I) around ROI and one for measuring the incident light at the top of the canopy $\left(I_{0}\right)$ (Figure 3). The measurement period was from $12^{\text {th }}$ June to $30^{\text {th }}$ September, 2019, and the photosynthetic photon flux density (PPFD) was measured every one minute. The data measured from 7:00 to 18:00 was used for this analysis. In addition, PPFD measurements of six DEFI2-L under the same sunlight condition were also performed for about 1 week before and after the field measurement for calibration between the sensors.
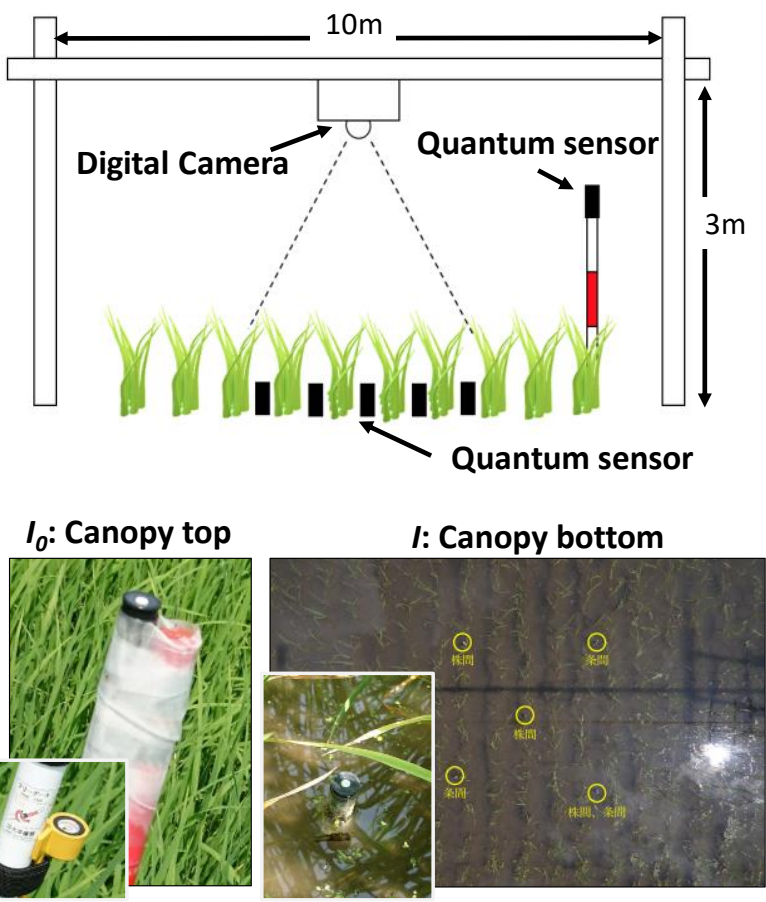

Figure 3. PPFD measurement at the top and bottom of canopy 
As a parameter of the light environment within the canopy, the relative PPFDs $\left(I / I_{0}\right)$ at five places were calculated from dividing the PPFDs at the canopy bottom by the PPFD at the canopy top. That is, the relative PPFD shows the transmissivity within the canopy. The instantaneous incident PPFD and transparent PPFD should be drastically changing because of cloud movements, but it possible to use them as the daily relative PPFD by averaging the diurnal data. In addition, the absorption coefficient: $\mathrm{K}$ was derived from actual LAI $\left(\mathrm{m}^{2} / \mathrm{m}^{2}\right)$ measured using automatic area measurer (AAM-9, Hayashi Denko) and relative PPFD measured under the same condition in another field, and this absorption coefficient $(\mathrm{K}=0.568)$ was applied to calculate the LAI by eq. 4 of canopy photosynthesis model (Monshi, Saeki, 1953) using relative PPFDs measured in our field.

$$
\frac{I}{I_{0}}=e^{-k \cdot L A I}
$$

\section{RESULTS AND DISCUSSION}

\subsection{Time series changes}

To understand the seasonal patterns of each CIs, VC, PH and relative PPFD / LAI during planted period, time-series changes are shown as follows.

\subsubsection{Daily CIs and VC}

Daily changes of gratio (as one example of CIs) and $V C$ in ROI are shown in Figure 4. $V C$ shows smoothly and stably change by comparing with gratio and the maximum value of about 0.6 around the heading date. Gratio begins to increase near the maximum tiller stage and gradually decreased after the heading date. The reason why two values decreased after the heading period was changing the color of the heads from green to yellow, and it might be difficult to distinguish green leaves and headings.

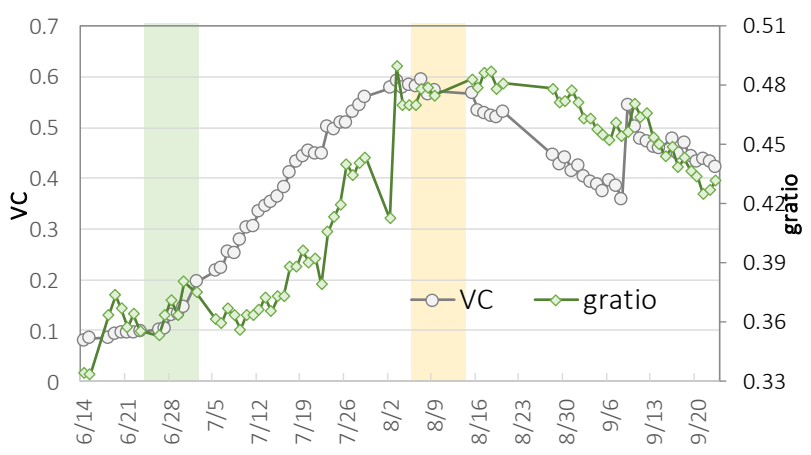

Figure 4. Daily changes of gratio and VC (light green: maximum tiller stage and light orange: heading date)

\subsubsection{Weekly PH}

Figure 5 shows weekly change of averaged $P H$ in ROI from $4^{\text {th }}$ June ( 2 weeks after transplanting). $P H$ does not increase to less than $10 \mathrm{~cm}$ by $9^{\text {th }} \mathrm{July}$, but has grown to nearly $30 \mathrm{~cm}$ in one week. However, the growth rate of $P H$ is not so high for the next 3 weeks (only $10 \mathrm{~cm}$ increase) until $6^{\text {th }}$ August. After that, the growth rate becomes high again, and $P H$ increases by about 40 $\mathrm{cm}$ in 3 weeks. The weather in 2019 was different with average year. According to the meteorological data at Fuchu, Tokyo by Japan Meteorological Agency, the temperature was lower and the sunshine duration was decreased about $80 \%$ in July by comparing with the data in 2018. However, the time-series changes of gratio and $V C$ (Figure 4) do not show the similar changing tendency with $P H$.
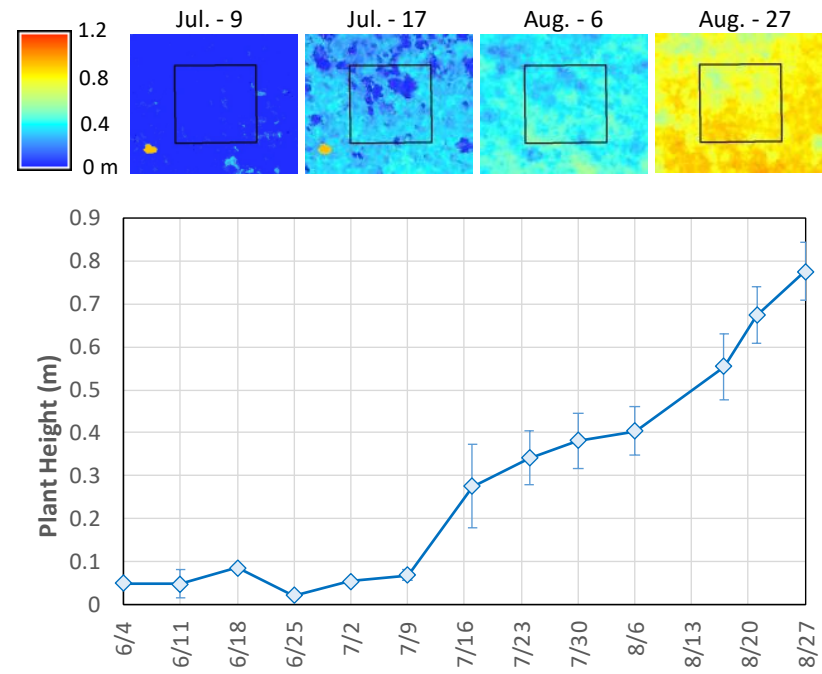

Figure 5. Weekly change of $P H$ (lower) and part of CSM in ROI on 4 days (upper)

3.1.3 Daily relative PPFD and LAI

Daily relative PPFD averaged at five points and LAI calculated from relative PPFD are shown in Figure 6. Relative PPFD begins to decrease from the maximum tiller period, and does not show any remarkable change until $14^{\text {th }}$ August until the heading date. Contrary to the relative PPFD, LAI begins to increase from around $1.0\left(\mathrm{~m}^{2} / \mathrm{m}^{2}\right)$ after the maximum tiller period, and no big change is observed after the heading date. On the other hand, $P H$ begins to increase after the maximum tiller period and continued to increase even after the heading period. Therefore, LAI calculated from relative PPFD has still increased a little even after heading. However, it is hard to understand the similar characteristics in relative PPFD change by comparing with the change of $P H$ (Figure 5).

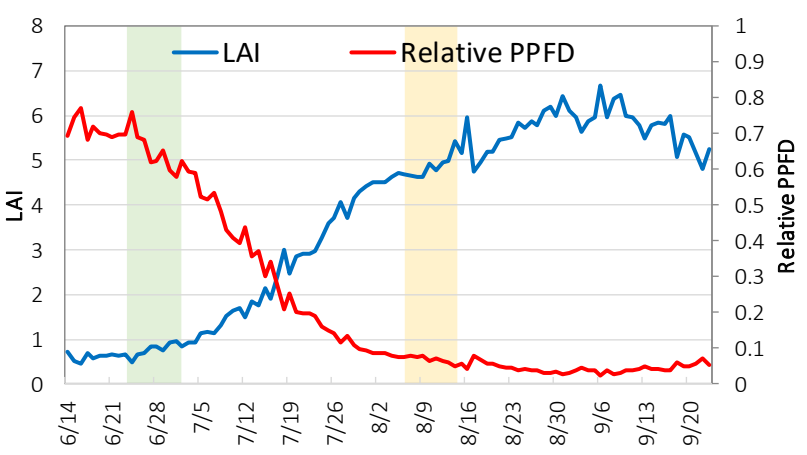

Figure 6. Daily change of relative PPFD and LAI (light green: maximum tiller stage and light orange: heading date)

\subsection{Relations of relative PPFD and LAI with CIs, VC and PH}

To clarify the relations of relative PPFD and LAI with all parameters of CIs, $V C$ and $P H$, the scattered graphs are shown in Figure 7-9. 
As for $V C$ and CIs, the data were used from $14^{\text {th }}$ June to $9^{\text {th }}$ August just before heading $(n=47)$, because the values decreased after heading. All relations of relative PPFD with three CIs and $V C$ (Figure.7) are expressed by linear regression with high determination coefficient: $\mathrm{R}^{2}$. Among them, $V C$ does not show a large variation in the value from the tiller stage to the heading date after transplantation, and had the highest $\mathrm{R}^{2}$. Although the $\mathrm{R}^{2}$ of gratio is high, the value varies around near heading date. On the contrary, VIgreen and GR show the variation around the tiller stage. The gratio is the ratio of $\mathrm{G}$ to the total RGB value, the VIgreen and $G R$ are the normalized index or ratio of $\mathrm{G}$ to $\mathrm{R}$, and $\mathrm{B}$ is not considered. From this, it is considered that the influence of the water surface at the early stage of growth influences the dispersion of values in VIgreen and GR.

Figure 8 shows the relations of the calculated LAI with gratio, which has the highest $\mathrm{R}^{2}$ in CIs, and $V C$. Linear regression was obtained for gratio, while $V C$ was exponentially regression. However, when the gratio is around 0.47, the LAI is distributed over a wide range of 1.9 to 4.3 , which means that it is difficult to explain when LAI is around 2 or above. On the other hand, in $V C$, the LAI is distributed in the range of 3.1 to 4.3 around the maximum value of 0.6 , and the LAI up to 3.5 is well explained.

According to the equation of canopy photosynthesis model based on Lambert - Beer law, the relation of LAI with gratio basically should be expressed as exponential function because the relation of relative PPFD with gratio shown in linear regression (Figure 7). This result also suggests that $V C$ is more suitable than gratio as a parameter in the relative PPFD and LAI estimation models.

As for the comparison with $\mathrm{PH}$, we used the data from $11^{\text {th }}$ June to the $27^{\text {th }}$ August $(\mathrm{n}=12)$. The relations of relative PPFD and LAI with $P H$ are expressed as the exponential and linear functions respectively (Figure 9). From the Napier's coefficient of -4.38 , that means, the transmissivity is attenuated to about $1.3 \%$ when the $P H$ reaches 1 meter, and this coefficient corresponds to optical depth within the canopy. In addition, since LAI is shown as a linear function of $P H$, it is clear that a suitable relational expression was obtained as mentioned before.
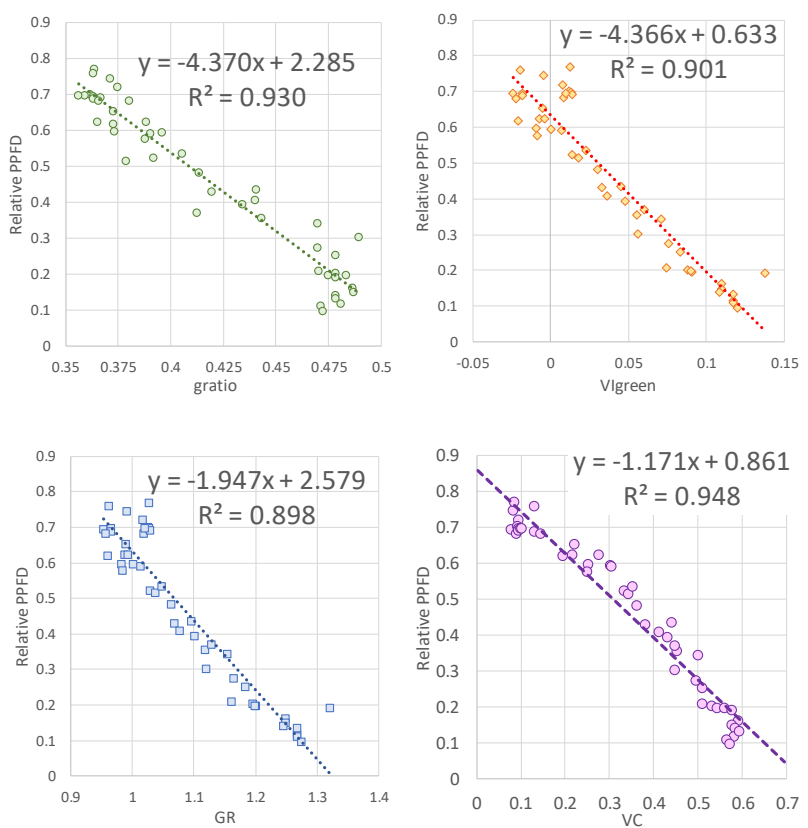

Figure 7. Relations of relative PPFD with gratio, VIgreen, $G R$ and $V C$
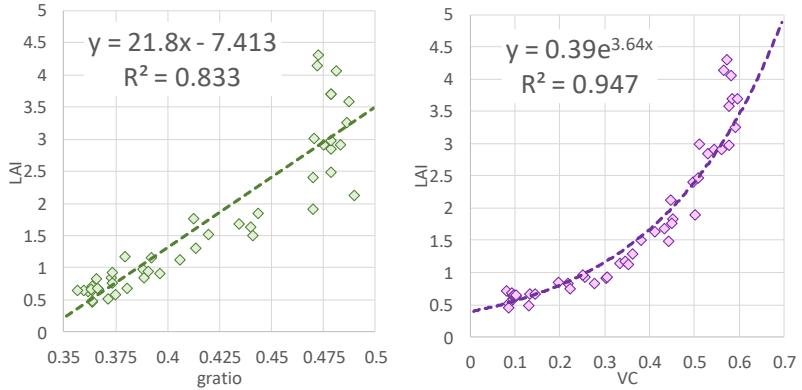

Figure 8. Relations of LAI with gratio and $V C$
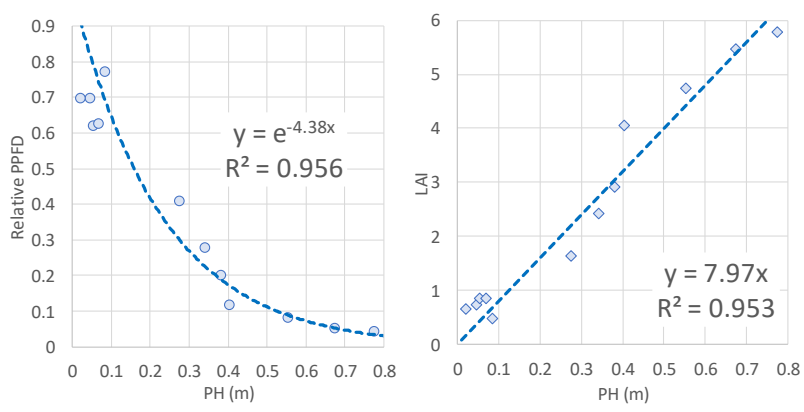

Figure 9. Relations of relative PPFD and LAI with $P H$

\subsection{Possibility of spatio-temporal monitoring}

From the above results based on our observations, it was shown that $V C$ and $P H$ are more effective than CIs as parameters for estimation models of relative PPFD and LAI. In order to understand the distribution of relative PPFD and LAI spatially and temporally, these relational functions shown in Figures 7-9 might be applied to $V C$ and $P H$ in whole planted area calculated from the ortho mosaic visible images and CSMs. However, we conducted these kinds of measurements for one variety and at one field only in this study, so that the obtained data was not enough to derive the efficient models and to evaluate the models. For development of robust model to estimate LAI, it would be necessary to collect the observational data under different field and weather conditions.

\section{CONCLUDION}

We conducted the observations to capture the RGB digital camera images using fixed camera and UAV and to measure the light environment within the rice canopy in order to clarify the relations of relative PPFD and LAI with several parameters can be obtained by ground- and UAV-based remote sensing methods. We used some of CIs and $V C$ calculated from RGB images taken by fixed camera every day and $P H$ generated from overlapped aerial images taken by UAV every week. As the results in this study, we confirmed that $V C$ and $P H$ would be effective to use the parameters for relative PPFD and LAI monitoring spatiotemporally.

\section{ACKNOWLEDGEMENTS}

This research was supported partially by a Grant-in-Aid for scientific research (16K07969) from the Ministry of Education, Science and Culture, Japan. 


\section{REFERENCES}

Aasen H., Honkavaara E., Lucieer A., Zarco-Tejada P., 2018: Quantitative remote sensing at ultra-high resolution with UAV spectroscopy: A review of sensor technology, measurement procedures, and data correction workflows. Remote Sensing, 10: 1098.

Cen H., Wan L., Zhu J., Li Y., Li X., Zhu Y., Weng H., 2019: Dynamic monitoring of biomass of rice under different nitrogen treatments using a lightweight UAV with dual image-frame snapshot cameras. Plant Methods, 15:32.

Gitelson, A. A., Kaufman, Y. J., Stark, R., and Rundquist, D., 2002: Novel algorithms for remote estimation of vegetation fraction. Remote Sensing of Environment, 80: 76-87.

Honkavaara, E., Heikki S., Jere K., Ilkka P., Teemu H., Paula L., Jussi M., and Liisa P., 2013: Processing and Assessment of Spectrometric, Stereoscopic Imagery Collected Using a Lightweight UAV Spectral Camera for Precision Agriculture. Remote Sensing 5(10):5006-39.

Li, S., Fei Y., Syed T. A., Zheng, H., Cheng, T., Liu, X., Tian, Y., Zhu, Y., Cao, W., and Cao, Q., 2019: Combining Color Indices and Textures of UAV-Based Digital Imagery for Rice LAI Estimation. Remote Sensing 11(15).

Maes, W. H. and Steppe, K., 2019: Perspectives for Remote Sensing with Unmanned Aerial Vehicles in Precision Agriculture. Trends in Plant Science 24(2):152-64.

Monsi, M., and Saeki, T., 1953: Über den Lichtfaktor in den Pflanzengesellschaften und seine Bedeutung für die Stoffproduktion. Japanese Journal of Botany 14: 22-52.

Shimojima, K., Ogawa, S., Naito, H., Valencia, M. O., Shimizu, Y., Hosoi, F., Uga, Y., Ishitani, M., Selvaraj, M. G., Omasa, K., 2017: Comparison between Rice Plant Traits and Color Indices Calculated from UAV Remote Sensing Images. Eco-Engineering, 29 (1): 11-16.

Wang Y., Wang D., Shi P., Omasa K., 2014: Estimating rice chlorophyll content and leaf nitrogen concentration with a digital still color camera under natural light. Plant Methods, 10:36

Yamashita M., Shinomiya Y., Yoshimura M., 2019: Development of methodology for plant phenology monitoring by ground-based observation using digital camera. ISPRS Ann. Photogramm. Remote Sens. Spat. Inf. Sci. IV-3/W1: 65-70. 